\title{
Preparation and characterizations of glyceryl oleate ufasomes of terbinafine hydrochloride: a novel approach to trigger Candida albicans fungal infection
}

\author{
Sankha Bhattacharya (D)
}

\begin{abstract}
Background: Worldwide fungal infection cases are increasing by leaps and bounds. The patients who are immunocompromised, i.e., cancer and AIDS, are more susceptible to different types of fungal infections like cutaneous candidiasis and its associate infections. The available treatment for such a disease is creams, gels, etc. However, due to the lack of penetrability and higher systematic absorption, these formulations have reported many side effects. To overcome such challenges, various novel drug delivery systems were introduced. The present research focused on the preparation of glyceryl oleate ufasomes of terbinafine hydrochloride using the film hydration method.

Result: The prepared formulations were characterized for globular size $(\mathrm{nm})$, zeta potential (mV), PDI, morphological characteristics, thermal behavior, in vitro drug release, in vitro antifungal activity, and in vitro skin permeation retention studies. After suitable formulation optimization using thin-film hydration method, 3:7 drug to glyceryl oleate ratio, UF3 formulation was found to produce higher drug entrapment efficacy $(52.45 \pm 0.56 \%)$, stable anionic zeta potential ($33.37 \pm 0.231 \mathrm{mV}$ ), desired globular size $(376.5 \pm 0.42 \mathrm{~nm})$, and decent polydispersity index (0.348 \pm 0.0345$)$. Diffusioncontrolled and zero-order sustained release profile was observed in the optimized UF3 batch. From the 5 days in vitro antifungal activity studies, it confirmed that UF3 ufasomes possessed good applicability in more prolonged therapy.

Conclusion: From the current investigation, it can be concluded that glyceryl oleate ufasomes of terbinafine hydrochloride could be an excellent approach to treat topical fungal infections.
\end{abstract}

Keywords: Antifungal agent, Cutaneous candidiasis, Glyceryl oleate, In vitro antifungal activity, Terbinafine hydrochloride, Ufasomes

\section{Highlights}

- Due to the recent elevation in the number of fungus infections and incapability of current medicaments, it is necessary to prepare a novel drug delivery system to circumvent all challenges.

- In this research work, terbinafine hydrochloride ufasomes were made using glyceryl oleate.

Correspondence: sankhabhatt@gmail.com

Department of Pharmaceutics, School of Pharmacy \& Technology

Management, SVKM'S NMIMS Deemed-to-be University, Shirpur, Maharashtra 425405, India

Springer Open
- Prepared ufasomes were characterized for globular size, zeta potential, PDI, morphological characteristics, thermal behavior, in vitro drug release, and in vitro skin permeation retention studies.

- Skin retention of vesicular dispersion was studied in Strat- $\mathrm{M}^{\circ}$ membranes.

- In vitro antifungal activity was performed in Candida albicans-infected male guinea pig.

- UF3 formulation was found to be the optimized formulation with best skin penetration and longer retention potential

(c) The Author(s). 2020 Open Access This article is licensed under a Creative Commons Attribution 4.0 International License, which permits use, sharing, adaptation, distribution and reproduction in any medium or format, as long as you give appropriate credit to the original author(s) and the source, provide a link to the Creative Commons licence, and indicate if changes were made. The images or other third party material in this article are included in the article's Creative Commons licence, unless indicated otherwise in a credit line to the material. If material is not included in the article's Creative Commons licence and your intended use is not permitted by statutory regulation or exceeds the permitted use, you will need to obtain permission directly from the copyright holder. To view a copy of this licence, visit http://creativecommons.org/licenses/by/4.0/. 


\section{Background}

The skin is the outermost protective layer of the human body, which protects internal organs from any kind of external damage [1]. The outer layer of skin is called the epidermis, which is responsible for skin tone. The next layer is the dermis, which is entirely comprised of hair follicles, connective tissues, and sweat glands [2]. The hypodermis or subcutaneous tissues are the deeper layers of the muscles [3]. The bacteria or fungus eventually do some transcutaneous invasion. To avoid such conditions, the outer epidermis acts as a protective barrier [4]. However, the skin permeability could be dogged when the fungus attacks the stratum corneum and ultimately alter the permeability of the skin [5]. The recent research findings are suggesting that more than one billion people of our planet have skin infections. Those patients who are immunocompromised are suspectable for such kinds of skin infections [6]. Mostly, due to the consumption of immunocompromised drugs or diseases like AIDS, leukemia, viral hepatitis, and multiple myeloma, the risk of fungal infections increased in the modern age [7]. Contemporary fungal infections can be virulent and invasive and can spread from subcutaneous to epidermis [8]. To target such fungal infections, it is necessary to have an excellent permeation enhancer. To enhance skin permeation, glyceryl oleate is one of the finest permeation enhancers for skin, which is capable to produce deep penetration. Due to the subcutaneous fluidization and polar lipid existing in the various liquid crystal phase, the amphiphilic glyceryl oleate shows higher penetrability into the skin [9]. The different types of essential fatty acids, such as alphalinolenic acid, linoleic acid, arachidonic acid, and oleic acid, are capable of producing vesicular structure in aqueous conditions [10]. Barreto et al. [11], in their study, prepared garlic liposomes with oleic acid and phosphatidylcholine and checked its antifungal activity in wheat bread [12]. From the thermal gravimetry analysis, it was concluded that oleic acid retards thermal decomposition as compared with phosphatidylcholine liposomes. In another research, Srisuk et al. performed an in vitro permeability study of methotrexate liposomes made up with oleic acid and phosphatidylcholine, which was further compared with liposomes of phosphatidylcholine and cholesterol [13]. The result of this research revealed that liposomes made up of phosphatidylcholine and oleic acid enhanced the skin permeation; hence, maximum methotrexate flux was witnessed in dermis layers of porcine skin. In another research, Sharma et al. has prepared potential fatty acid vesicle comprising with oleic acid for the topical drug delivery of methotrexate [14]. In another research, Salama et al. developed unsaturated fatty acid liposomes or ufasomes using oleic acid [15]. In another study, Sharma and Arora [14] encapsulated methotrexate in oleic acid vesicle to form ufasomes for the treatment of psoriasis; the results were astonishing. The oleic acid methotrexate ufasomes have had maximum entrapment efficacy in rat skin as compared with carbopol-based plain gel [16].

Terbinafine hydrochloride, an allylamine derivative structurally related to naftifine, can block the biosynthesis of ergosterol by suppressing squalene epoxide [17]. This drug can be used to treat different types of skin infections caused by fungus. Terbinafine is highly active against dermatophytes [18]. Eventually, this drug gel and creams are available in the market but how much they are bioavailable or penetrable is still a matter of research [19]. Moreover, some patients often withdraw medication once they are receiving a positive response from it, which ultimately increases the chance of reoccurrence of fungus infections in patients. Moreover, frequent dosing of terbinafine in the skin could increase the likelihood of higher absorption in systematic circulation [20].

As far as fatty acid vesicles are concerned, they can spontaneously grow in alkaline micelles, which are attached to buffer vesicles [21]. These fatty acid vesicles are capable of fusogenic characteristics, which ultimately lead to a reduction of the membrane transition temperature. When fatty vesicles come with the contacts of lipid bilayers of skin, it releases its contents [22]. Therefore, it is established by many types of research that fatty acid vesicles are the most effective carrier to boost skin penetrability through the stratum corneum [23].

In our present study, an attempt was made to prepare effective fatty acid vesicular drug delivery systems or ufasomes of terbinafine hydrochloride using glyceryl oleate for aiming fungus infections which affect the deeper epidermal layer of the skin. This formulation is capable of providing local effects with higher penetrability and reduced dose and toxicity. For preparing glyceryl oleate ufasomes of terbinafine hydrochloride, film hydration methods were used. The different characterization parameters were also addressed in this research.

\section{Methods \\ Materials}

Terbinafine hydrochloride was a gifted sample by Dr. Reddy's Laboratories (Ameerpet, Hyderabad, Telangana, India), glyceryl tri(oleate- $1-{ }^{13} \mathrm{C}$ ) was purchased from Sigma-Aldrich (Bangalore, India), Sephadex ${ }^{\circ}$ G-50 was purchased from Sigma-Aldrich (Bangalore, India), and dialysis tubing cellulose membrane with flat width 10 $\mathrm{mm}(0.4 \mathrm{in}$.) was purchased from Sigma-Aldrich (Bangalore, India). The fungal strain Candida albicans (Robin) 
Berkhout $\left(\right.$ ATCC $^{\circ} 10231^{\text {mw }}$ ) was a gift sample from RK University (Rajkot, India), synthetics non-human skin called Strat- $\mathrm{M}^{\odot}$ membrane arranged from Millipore (Canada), and the rest of the samples were analytical grades and purchased from Rankem Laboratories (Gurugram, Haryana, India)

\section{Preparation of terbinafine hydrochloride ufasomes}

By using a thin-film hydration method, the terbinafine hydrochloride ufasomes were prepared in a round bottom flask. After slide modification of the Sharma et al. [14] procedure, new preparation steps were incorporated [24]. Initially, terbinafine hydrochloride and glyceryl oleate were dissolved in dichloromethane and kept in a round-bottomed flask for vacuum drying using a rotatory evaporator (IKA-RV 3 V-C Digital Rotatory Evaporator, Germany). The assembly was set for $50 \mathrm{rpm}$ at 45 ${ }^{\circ} \mathrm{C}$. After overnight vacuum drying, a dried thin film formed, indicating complete removal of residual solvent. The thin film was further hydrated for $12 \mathrm{~h}$ with freshly prepared pH 5.5 phosphate buffer solution. The mixture was further sonicated in $1.5 \mathrm{~L}$ Athena Ultrasonic Bath Sonicator for $15 \mathrm{~min}$ to form uniform vesicular dispersion. Also, optimization is performed by changing the ratios of terbinafine hydrochloride and glyceryl oleate. The manual optimization procedure followed, where different ratios of terbinafine hydrochloride and glyceryl oleate were screen for the best optimization batch. A total of five (UF1-UF5) formulations batches was prepared, and subsequently, the best formulation batch has been selected after suitable statistical screening ( $p<$ 0.05). The unentrapped drug was separated from the formulations using OMNISEC Gel permeation Chromatography (Malvern Panalytical, UK), considering borate buffer as eluent.

\section{Vesicular size, zeta potential, and morphological characterization of glyceryl oleate vesicles}

Delsa Nano C Particle Size and Zeta Potential Analyzer (Beckman Coulter, USA) was used for accurate vesicular size and zeta potential measurement [25]. For measuring the internal morphology and surface characterization of the vesicles, transmission electron microscopy (JEOL Transmission Electron Microscopes, USA) and mitotic compound microscope (BA210 LE, Germany) were used.

\section{Entrapment efficacy}

Entrapment efficacy was measured after exposing the vesicles in $1 \mathrm{~N}$ sodium hydroxide [26]. The release of the terbinafine hydrochloride was measured spectrophotometrically (UV-1280-UV-VIS Spectrophotometer, Shimadzu Life Science, Japan) at $283 \mathrm{~nm}$. The following equation measured the entrapment efficacy:
Entrapment $(\%)=$ Entrapped drug/Total drug incorporated $\times 100$

\section{Differential scanning electron microscopy}

To understand the thermal behavior of drug, drugloaded vesicles, and blank vesicles, differential scanning electron microscopy was performed (Q-10, TA instrument) [22]. Calibration of heat flow is compulsory before initializing the process. Sample purging was done using inert nitrogen gas at a flow rate of $30 \mathrm{ml} / \mathrm{min}$. The temperature was raised at $20{ }^{\circ} \mathrm{C} / \mathrm{min}$ increments.

\section{In vitro drug release studies}

The dialysis tubing cellulose membrane with flat width $10 \mathrm{~mm}$ (0.4 in) was used for the in vitro dialysis of glyceryl oleate ufasomes. For proper execution of this study, $5 \mathrm{~mL}$ glyceryl oleate ufasomes were taken into dialysis tubing and both ends were tied using nylon thread and dispersed into a conical flask comprising of $50 \mathrm{~mL} \mathrm{pH}$ 5.5 phosphate buffer solution containing $0.01 \%$ sodium lauryl sulfate. The conical flask was further incubated into an incubated shaker for $15 \mathrm{~min}$ in $37^{\circ} \mathrm{C}$ at $60 \mathrm{rpm}$. Also, a 5-mL sample was withdrawn from the bottle, and $5 \mathrm{~mL}$ of fresh buffer was added into the conical flask. The withdrawn sample was filtered and determined spectrophotometrically at $283 \mathrm{~nm}$.

\section{In vitro antifungal activity of glyceryl oleate ufasomes}

The cup plate method was incorporated to estimate the antifungal activity of glyceryl oleate ufasomes. Initially, Candida albicans was first grown overnight and inoculated into Sabouraud dextrose agar (SDA) media plates. Using cork borer, 10-mm diameter, three wells were introduced after solidification of Candida albicans in media plates. In the first good glyceryl oleate, $30 \% \mathrm{w} / \mathrm{w}$ ufasomes was placed as a test group; in the second well, plain $2 \% \mathrm{w} / \mathrm{w}$ terbinafine hydrochloride was placed in third $\mathrm{pH} 5.5$ phosphate buffer solution was placed, which is considered a controlled group. The plates were incubated for $48 \mathrm{~h}$ at $37^{\circ} \mathrm{C}$. At different time intervals, the zone of inhibition was measured for the control, plan drug, and test sample.

\section{In vitro skin permeation study for glyceryl oleate ufasomes}

Using Franz diffusion cell (Dolphin Pharmacy Instruments Private Limited, India) in vitro skin permeation study for glyceryl oleate ufasomes (sample A), marketed gel (B-Fine, Cian Healthcare Limited, Mumbai, India) and plain drug of terbinafine hydrochloride (gift sample, Dr. Reddy's Laboratories) were carried out. The diffusion area for the Franz diffusion cell was $4.5 \mathrm{~cm}^{2}$, and the 60$\mathrm{mL}$ volume was kept inside. The penetration study was done using a synthetic non-animal-based model called 
$47 \mathrm{~mm}$ 60/pk Strat-M membrane [27] (Millipore, Canada), which is specifically designed to mimic human skin. The most significant advantages of this synthetic skin are no need for an animal model, deficient human to animal skin variability alternative $(\mathrm{CV}=8 \%)$, almost around 1.38 correlation to human skin, and longer selflife with no specific storage requirement. In the experimental procedure, the disk-shaped Strat- $\mathrm{M}^{\circ}$ membrane was plucked using forceps and socked into a Petri dish, continuing $10 \mathrm{~mL}$ of saline water.

Further, the Strat- $\mathrm{M}^{\bullet}$ membrane was placed into the donner compartment of the Franz diffusion cell using forceps and maintain temperature around $35-37^{\circ} \mathrm{C}$. Thirty percent $\mathrm{w} / \mathrm{w}$ optimized glyceryl oleate ufasomes, $1 \% \mathrm{w} / \mathrm{w}$ B-Fine Marketed gel (2.91 mg equivalent of terbinafine hydrochloride), and $2.91 \mathrm{mg}$ of terbinafine hydrochloride were placed in the donner compartment of three different Franz diffusion cell. Nearly $0.5 \mathrm{~mL}$ of samples were withdrawn from the acceptor compartments for various time alternatives within $24 \mathrm{~h}$ using digital micropipettes (Thermo Fisher Scientific India Private Limited, Mumbai, India). The same amount of buffer solutions was added to the maintained sink condition in the acceptor compartment. The filtered samples were analyzed using UV-1280-UV-VIS Spectrophotometer (Shimadzu Life Science, Japan) at $283 \mathrm{~nm}$.

\section{Skin retention of vesicular dispersion}

After performing in vitro skin permeation studies in three Strat- $\mathrm{M}^{\circ}$ membranes [28], the utilized membranes were carefully removed from the Franz diffusion cell and subsequently chopped into small pieces. Now, $50 \mathrm{~mL}$ of pH 5.5 methanolic phosphate buffer (6:4) was added into the cut pieces and placed in shakers incubators with Vortexes Stirrer (Athena Technology, Mumbai, India) for $1 \mathrm{~h}$ at $37{ }^{\circ} \mathrm{C}$. This procedure completely extracts drug from the embedded Strat- $\mathrm{M}^{\circ}$ membrane. After extraction, the resultant solutions were filtered using a compact cooling centrifuge (Remi Elektrotechnik Limited, Mumbai, India) and using UV-1280-UV-VIS Spectrophotometer at $283 \mathrm{~nm}$ the embedded amount of drug estimated.

The skin retention was calculated using the following formula:

Skin retention $(\%)=\frac{\text { amount of drug embedded in Strat }-M^{\circ} \text { Membranes }}{\text { Total drug incorporated }} \times 100$

\section{In vivo studies}

While performing animal studies, the Committee for Control and Supervision of Experiments on Animals (CPCSEA) guidelines issued by the Government of India followed. The animal ethical committee approved all the study protocol of ISF College of Pharmacy, Moga, Punjab, India.

\section{In vivo antifungal activity of gel}

In developing a model of immunocompromised animals, $5 \mathrm{mg} / \mathrm{kg} /$ bodyweight of Cyclosporine, a calcineurin Inhibitor, the injection was administered subcutaneously in 450-500 g weighted male Guinea pig. Cyclosporine was applied five times, i.e., 3 days before the injection of fungal suspension and 2 days after the inoculation of the suspension.

\section{Preparation of Candida albicans inoculums}

Candida albicans (Robin) Berkhout (ATCC $10231^{\mathrm{TM}}$ ) was utilized to inoculate fungal infections in the Guinea pig. Initially, it is necessary to prepare a culture strain. For this purpose, sabouraud dextrose agar solution was used. The Candida albicans were inoculated in it for $48 \mathrm{~h}$ for $37^{\circ} \mathrm{C}$. The expressed cells were collected and resuspended in sterile saline. It was making sure that the final concentration in the sterile saline must be $10^{7}$ colony-forming unit $/ \mathrm{mL}(\mathrm{cfu} / \mathrm{mL})$

\section{Initiation of fungal infection in the skin}

Before shaving the animals back, it is necessary to anastasis the animal using 5-10 mg/k IM Ketamine. The back was shaved using NOVA Electric Trimmer Clipper. The total surface area trimmed was $3.0 \mathrm{~cm}^{2}$. Each animal body back surface area was inoculated with $10^{7} \mathrm{cfu} / \mathrm{mL}$ of Candida albicans dispersion using a sterile cotton swab (socked in $70 \% \mathrm{v} / \mathrm{v}$ ethanol). The swab was rubbed on the surface of anesthetized animals unless and until no fluid certain in the animal body surface. Further, animals were caged separately. Animals were observed from time to time, and the sign of intense erythema was investigated into the site on the animal body.

\section{Treatment of Candida albicans-induced infection}

Once the symptoms of infections were palpable, the treatment would begin. The animals were devised into four groups. Each group contains four animals. The first group was treated with marketed B-Fine gel, equivalent to $5.8 \mathrm{mg}$ of terbinafine hydrochloride (sample A); the second group was treated with terbinafine hydrochloride-loaded glyceryl oleate ufasomes, equivalent to $5.8 \mathrm{mg}$ of terbinafine hydrochloride (sample B); the third group was treated with $5.5 \mathrm{mg}$ of terbinafine hydrochloride, which was sprinkled on the surface of exposed skin (sample C); and finally the fourth group was exposed with the $\mathrm{pH} 5.5$ phosphate buffer solution, which is considered a controlled group (sample D). Before applying samples from A to D in animal skin, one animal from each group was sacrificed to check the initial colony count (day 0), as prescribed in Aggarwal and Goindi [29]. For the 3 alternative 
days, animals were ingested with treatment once in a day. For the measurement of the colony count, $24 \mathrm{~h}$ after the last treatment, each animal from each group was sacrificed (day 1), subsequently after $72 \mathrm{~h}$ (day 3 ) and $120 \mathrm{~h}$ (day 5); the same procedure was followed for each and every group. Further, the treatment skin was separated from the animal body and carefully soaked in saline solution and homogenized. An aliquoted portion of homogenate was placed in sabouraud dextrose agar (SDS) plats for $48 \mathrm{~h}$ at $37^{\circ} \mathrm{C}$, and colony-forming unit (cfu) value was recorded using digital colony counter (J. S. Enterprises, New Delhi, India).

\section{Statistical analysis}

Using analysis of variance (ANOVA), the treatment group was analyzed with a controlled group. The multiple comparison test of ANOVA was performed in GraphPad Prism v.7.0 (San Diego, Canada). All the results were expressed in mean $\pm \mathrm{SD}$. The $p$ value $<0.05$ was considered significant.

\section{Result \\ Morphological characterization of glyceryl oleate ufasomes of terbinafine hydrochloride}

The shape and morphology of ufasomes were determined by using motic compound microscope (BA210 LE, Germany) and transmission electron microscopy (JEOL Transmission Electron Microscopes, USA). The optimized ufasomes were stained using sulforhodamine B solution (sulforhodamine B solution previously prepared using $0.5 \%$ solutions of acetic acid and water) for the motic microscopic studies. The motic image of glyceryl oleate ufasomes of terbinafine hydrochloride was shown in Fig. 1a, and the TEM image of the same as shown in Fig. 1b. The two images clearly indicating prepared ufasomes were spherical in shape and size is below $500 \mathrm{~nm}$.
Globular size, percentage drug entrapment efficacy, and colloidal nature of ufasomes

Prepared ufasomes of terbinafine hydrochloride characterized by zeta potential, drug entrapment efficacy, polydispersity index, and particle size. Table 1 represents the process of optimization. In the process of optimization, it was observed that UF3 formulation was found to be statistically significant (The $p$ value $<0.05$ ) as compared to other formulations. From the Table 1, it is signifying that drug-glyceryl oleate 3:7 molar ratio of UF3 formulation possessed with optimum globular size $376.5 \pm 0.42$ nm (Fig. 2a) with more stable anionic zeta potential $33.37 \pm 0.231 \mathrm{mV}$ (Fig. 2b). Another interesting fact that is observed is the increased molar ratio of glyceryl oleate after UF3 formulation decreases the entrapment of the ufasomes. It is due to the increase in the concentration of drug in the bilayer of ufasomes. Moreover, the higher anionic zeta potential of UF4 and UF5 also would be the reason for instability in these formulations. Further addition of drugs could lead to instability within the ufasomes molecule, and subsequently leakage would be palpable. Since UF3 was showing excellent colloidal stability and least particle size among all the beaches, hence it could be anticipating that UF3 would have good skin penetration behavior.

\section{Differential scanning calorimetry (DSC)}

Thermal behavior and drug lipid conjugation can be identified using DSC analysis. The thermogram of the drug, blank ufasomes, and drug-loaded ufasomes was shown in Fig. 3. A sharp endothermic peak of terbinafine hydrochloride at $178.43^{\circ} \mathrm{C}$ indicating the melting of the drug. Most importantly, no characteristic drug peak was found in drug-loaded ufasomes of glyceryl oleate, meaning the drug was possibly encapsulated or entrapped in glyceryl oleate vesicles, which is justifying the higher entrapment efficacy of UF3-optimized formulation. It was

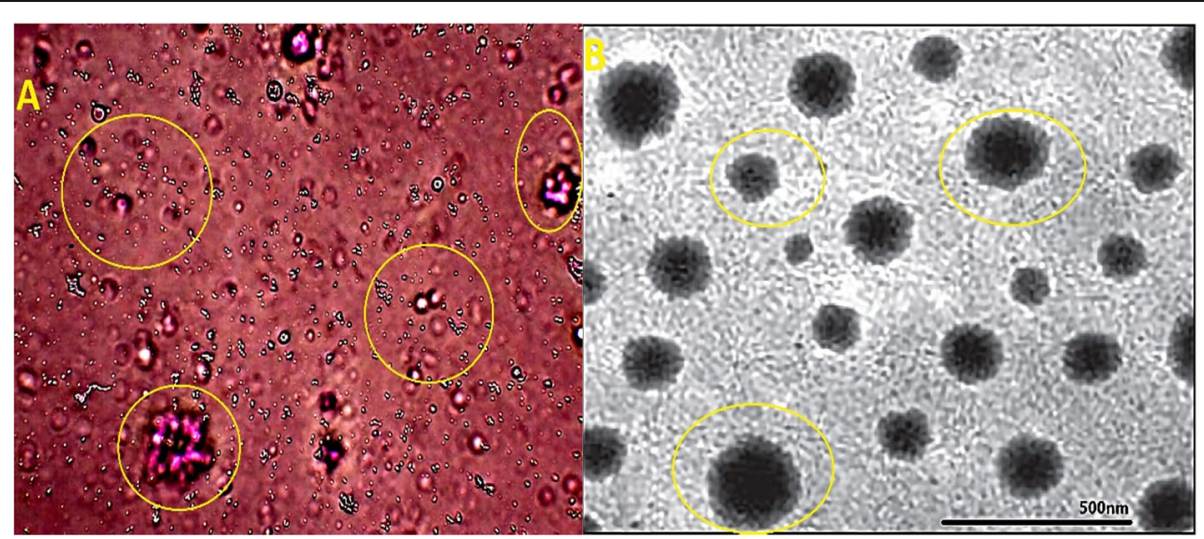

Fig. 1 a The yellow circles indicating the formation of ufasomes in the slide. $\mathbf{b}$ Transmission electron microscopy (TEM) image of glyceryl oleate ufasomes of terbinafine hydrochloride at $80 \mathrm{kV}$ and $\times 50,000$ 
Table 1 Optmization of ufasomes of terbinafine hydrochloride (where value represented in mean \pm SD $[n=3]$ )

\begin{tabular}{llllll}
\hline Formulation code & $\begin{array}{l}\text { Terbinafine hydrochloride } \\
\text { to glyceryl oleate }\end{array}$ & Globular size $(\mathbf{n m})$ & Zeta potential $(\mathbf{m V})$ & PDI & Drug entrapment (\%) \\
\hline UF1 & $5: 5$ & $610.1 \pm 0.37$ & $-26.58 \pm 0.32$ & $0.527 \pm 0.043$ & $45.29 \pm 0.27$ \\
UF2 & $4: 6$ & $542.3 \pm 0.22$ & $-28.52 \pm 0.21$ & $0.403 \pm 0.037$ & $48.28 \pm 0.18$ \\
UF3 & $\mathbf{3 : 7}$ & $\mathbf{3 7 6 . 5} \pm \mathbf{0 . 4 2}$ & $\mathbf{- 3 3 . 3 7 \pm 0 . 2 3 1}$ & $\mathbf{0 . 3 4 8} \pm \mathbf{0 . 0 3 4 5}$ & $\mathbf{5 2 . 4 5 \pm 0 . 5 6}$ \\
UF4 & $2: 8$ & $423.6 \pm 0.42$ & $-34.27 \pm 0.11$ & $0.427 \pm 0.0378$ & $42.56 \pm 0.35$ \\
UF5 & $1: 9$ & $510.4 \pm 0.29$ & $-38.21 \pm 0.45$ & $0.528 \pm 0.053$ & $38.67 \pm 0.05$ \\
\hline
\end{tabular}

also observed that melting enthalpy and peak intensity were changed marginally for UF1, UF2, UF4, and UF5 formulations; this could be due to the lower entrapment of the drug molecule within the formulation. However, no such shift was observed when optimized UF3 was compared with blank glyceryl oleate ufasomes, indicating highly stable formulation.

\section{In vitro drug release study}

Dialysis tubing cellulose membrane with flat width 10 $\mathrm{mm}$ (0.4 in.) used to carry out in vitro drug release study. The in vitro drug release profiling from UF1 to UF5 is mentioned in Fig. 4. The drug release profiles from different formulation batches, suggesting that a minimum $38.48 \%$ cumulative drug release was witnessed in UF2 formulation. Where else, a maximum $55.48 \% \mathrm{cu}-$ mulative drug release was seen in UF3-optimized formulation after $30 \mathrm{~h}$. The drug release kinetics is depicted in Table 2. Almost all the formulation was showing zeroorder release kinetics; since, the $R^{2}$ value in the zeroorder model was showing a maximum. The KorsmeyerPappas release exponential $(n)$ was leading the amount from 0.356 to 0.432 , which indicates diffusional release kinetics in UF1 to UF2 formulations. However, UF3 formulations have shown entirely zero-order kinetics $\left(R^{2}=\right.$ 0.9926). Nevertheless, from Fig. 4 , it is indicating that during $15-25 \mathrm{~h}$ of drug release, the formulations (UF1UF5) are showing maximum zero-order kinetics.

\section{In vitro antifungal activity of applied ufasomes in animal skin}

In Fig. 5, the antifungal activity of UF1 to UF5 was reported against Candida albicans. The zone of inhibition is not witnessed in the controlled group. However, it was noticed that a sharp increase in the zone of inhibition seen in plain terbinafine hydrochloride up to the 36th hours as compared to the ufasome formulations (UF1-UF5). But from the 48th hours onwards, the zone of inhibition decreased in plain drug; however, for almost all the ufasome formulations (UF1-UF5) a sharp increase in zone of inhibition perceived from the 48th hour. This is due to the diffusion-controlled release of the drug from the ufasomes surface after the 48th hour. Once again, UF3 was showing the highest zone of inhibition $(21.2 \mathrm{~mm})$ claimed to be an optimized formulation among all the formulations.
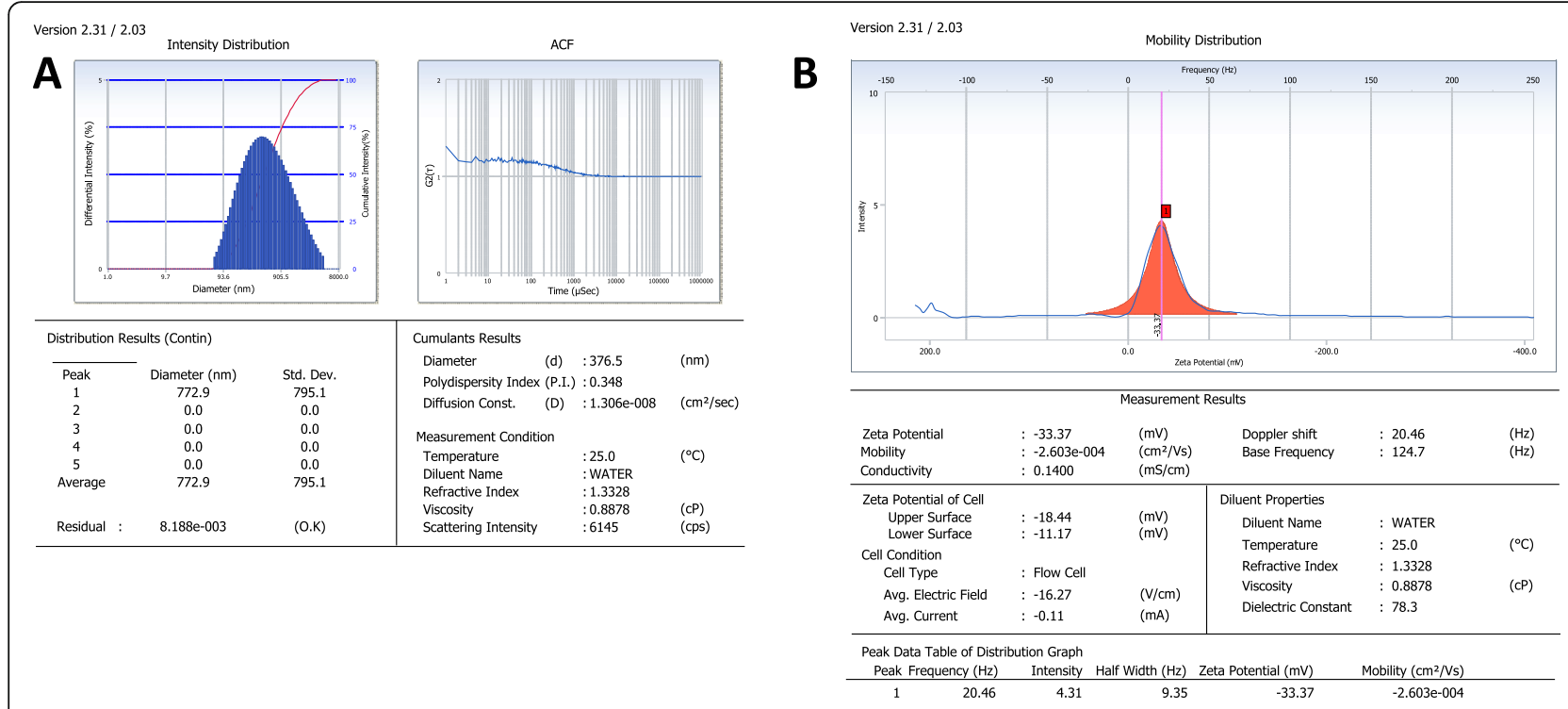

Fig. 2 a Globular size of optimized ufasomes (UF3). b Zeta potential of optimized ufasomes (UF3) 


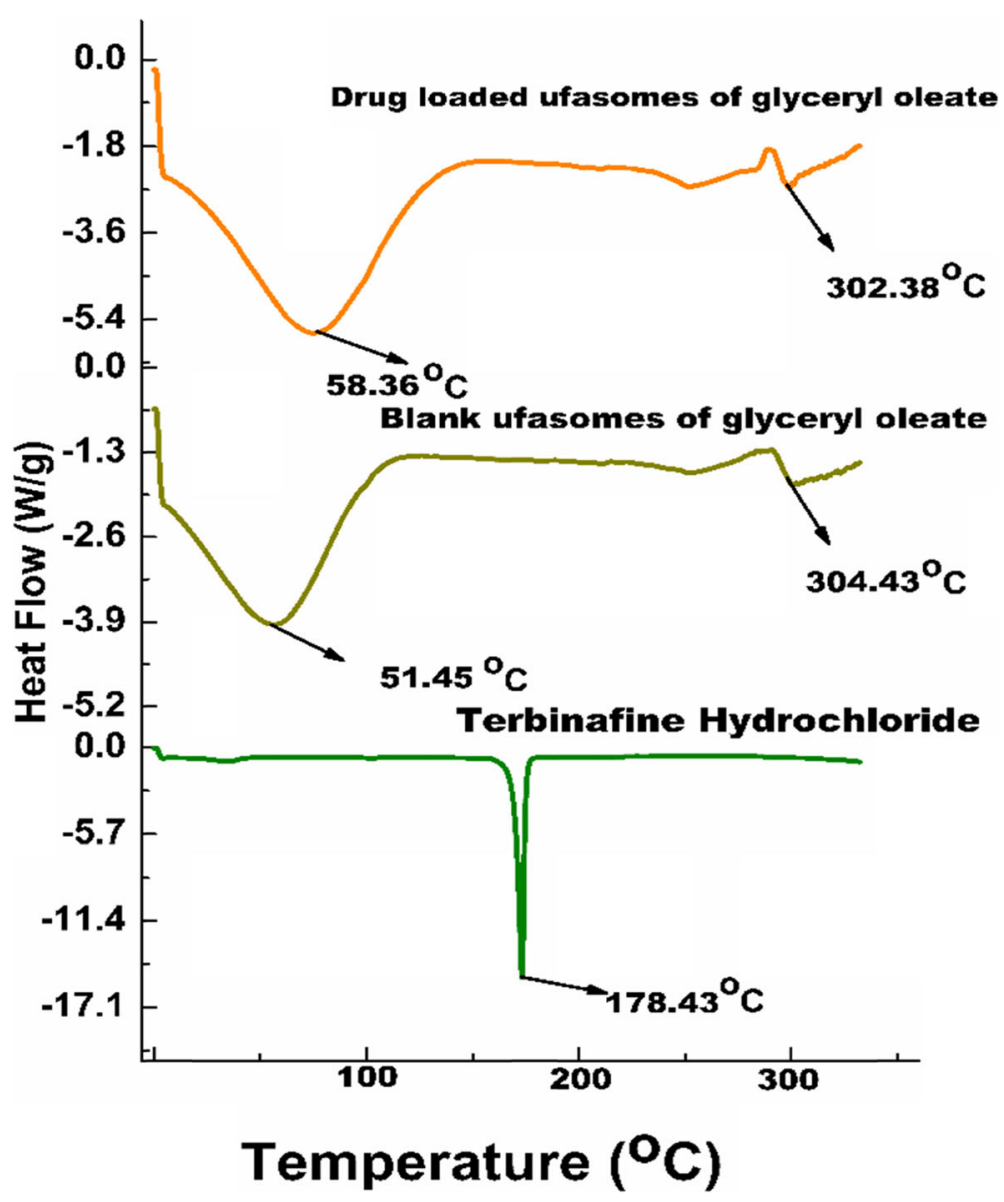

Fig. 3 DSC thermograph of terbinafine hydrochloride showing a sharp endothermic peak at $178.43^{\circ} \mathrm{C}$ (reported melting point $>202^{\circ} \mathrm{C}$ ), indicating melting of the drug, in case of blank glyceryl oleate ufasomes, apart from the sharp melting point at $51.45^{\circ} \mathrm{C}$; a less intense peak was observed in $304.43^{\circ} \mathrm{C}$; however, no characteristic drug peak was observed in drug-loaded ufasomes of glyceryl oleate

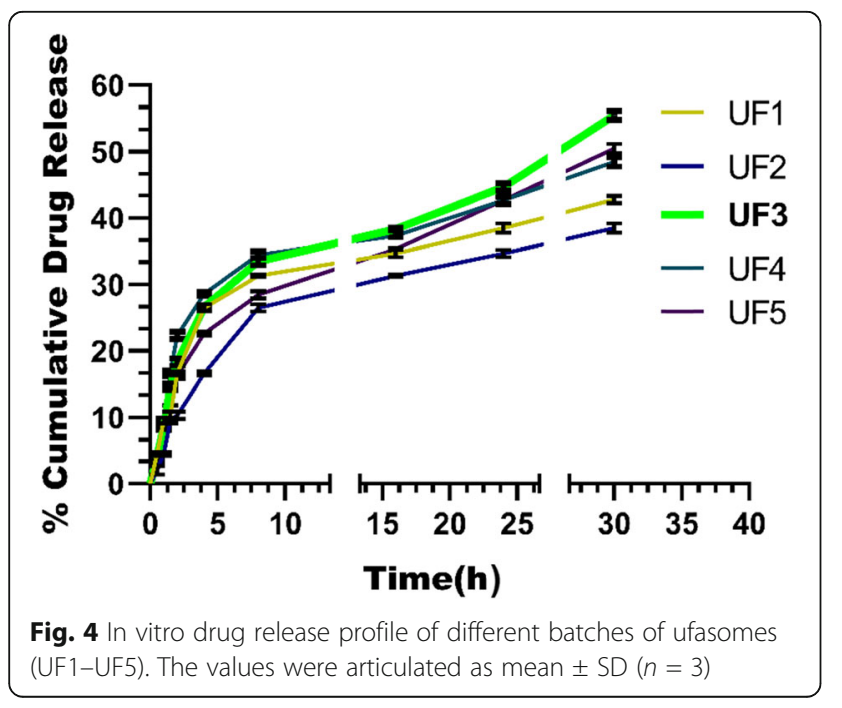

In vitro skin permeation of optimized UF3 ufasomes In vitro skin permeation studies were exercised on a non-animal-based model called $47 \mathrm{~mm}$ 60/pk Strat-M ${ }^{\odot}$ membrane (Millipore, Canada), which was specially designed to mimic human skin. The In vitro skin permeation study performed in Franz diffusion cell (Dolphin Pharmacy Instruments Private Limited, India). From Fig. 6 it is clear that UF3 formulation had a less penetration in Strat$\mathrm{M}^{\oplus}$ membrane compared to B-Fine-marketed formulation. The drug permeation of UF3 is less, i.e., $15.78 \pm 1.21 \%$ at the 24th hour; that means it has more retention index within the skin. The drug permeation of UF3 was low because the presence of glyceryl oleate in ufasomes increases the drug retention within the pours of Strat- $\mathrm{M}^{\bullet}$ membrane. The drug permeation from the B-Fine marketed cream is more because the formulation did not have any carrier system to hold the drug within. 
Table 2 Kinetic profiling of different ufasome formulation

\begin{tabular}{lllll}
\hline Ufasome formulation code & Higuchi $\left(\boldsymbol{R}^{\mathbf{2}}\right)$ & Zero-order $\left(\boldsymbol{R}^{\mathbf{2}}\right)$ & First-order $\left(\boldsymbol{R}^{\mathbf{2}}\right)$ & Korsmeyer-Pappas release exponential $(\boldsymbol{n})$ \\
\hline UF1 & 0.293 & 0.9638 & 0.943 & 0.356 \\
UF2 & 0.305 & 0.9561 & 0.9728 & 0.363 \\
UF3 & $\mathbf{0 . 3 5 6}$ & $\mathbf{0 . 9 9 2 6}$ & $\mathbf{0 . 9 8 6 4}$ & $\mathbf{0 . 4 3 2}$ \\
UF4 & 0.327 & 0.9835 & 0.9729 & 0.398 \\
UF5 & 0.305 & 0.9802 & 0.9629 & 0.374 \\
\hline
\end{tabular}

\section{Skin retention of UF3 formulation and B-fine marketed cream}

From the previous in vitro skin permeation experiment, it was confirmed that optimized glyceryl oleate ufasomes of terbinafine hydrochloride (UF3) possessed a drug reservoir effect in Strat- $\mathrm{M}^{\ominus}$ membrane. The percentage of terbinafine hydrochloride deposition was compared with B-Fine marketed formulation. The amount of drug retained in UF3 formulation in the 24th hour was $62.56 \pm 0.64 \%$, where else B-Fine marketed gel shows $14.52 \pm 1.43 \%$ in the same hour. Figure 7 showing skin retention studies.

In vitro antifungal activity studies against Candida albicans As described earlier, the in vitro antifungal activity of terbinafine hydrochloride, UF3, and B-Fine marketed formulation was determined by persuading Candida albicans based on infection in animal skin. From Fig. 8, it was indicated that UF3 has prominent antifungal activity against Candida albicans as compare to B-fine marketed formulation and terbinafine hydrochloride plain drug. At initial day 0 , almost all the groups were showing the same colony count $(6.25 \log \mathrm{cfu} / \mathrm{mL})$, which indirectly indicates that Candida albicans initialized fungus infections in the dermal layer of animal skin. Once the fungal progression was confirmed in the skin, the treatment process was ignited for successive days. On the 1st day, the controlled group was showing marginal growth in colony count (6.88 log cfu/ $\mathrm{mL}$ ), which is due to the propagation of Candida albicans in pH 5.5 phosphate buffer solution. On the other hand, due to the immediate release of terbinafine hydrochloride from the skin surface, on the first day, the plain drug (4.25 $\log \mathrm{cfu} / \mathrm{mL})$ and B-Fine marketed gel $(3.58 \log \mathrm{cfu} / \mathrm{mL})$ were showing good affectivity against Candida albicans. In the case of UF3 formulation, colony count was showing higher as compare to B-Fine gel in the 1st day (4.05 log cfu/ $\mathrm{mL}$ ). This phenomenon could be due to the lower amount of drug release from vesicular dispersion of UF3. The presence of glyceryl oleate in ufasomes retards the drug release. Further, after $72 \mathrm{~h}$ or the 3rd day, the colony count was started increasing drastically in plain drug (5.45-6.03 log $\mathrm{cfu} / \mathrm{mL}$ ), and B-Fine marketed-induced treatment (3.98$4.84 \log \mathrm{cfu} / \mathrm{mL}$ ), producing fewer significant statistics as

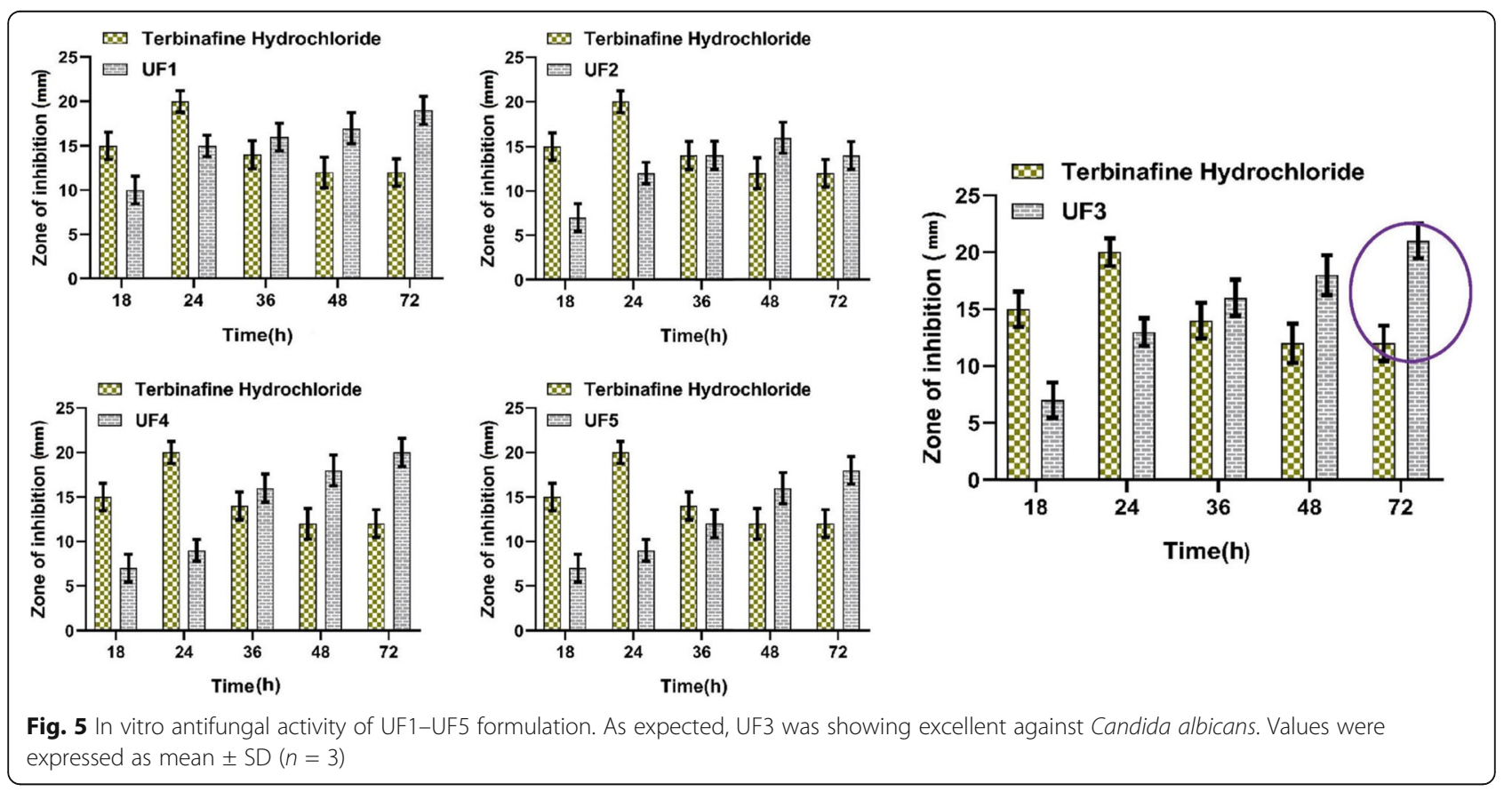




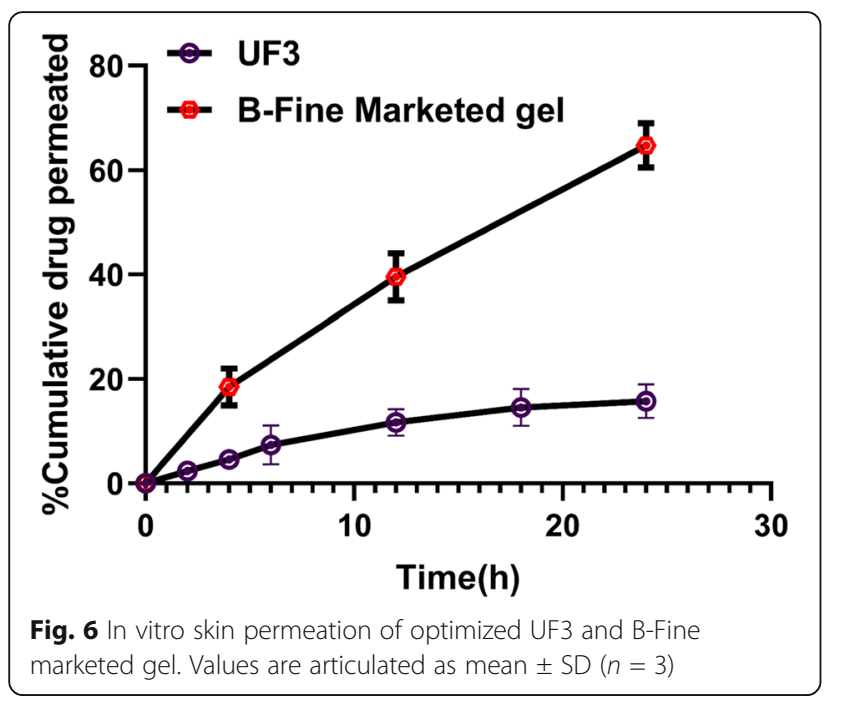

compared to the controlled group. The multiple comparison two-way ANOVA and Tukey test statistics were performed by GraphPad Prism 8.0 software. Super increase in colony count in plain drug and B-Fine marketed gel was due to the excessive release of the drug after the 1st day. Hence, no drug was available for further action $(p<0.05)$. In 5 th day, there is significant declined $(p<0.05)$ in colony count $(1.12 \log \mathrm{cfu} / \mathrm{mL})$ in UF3-treated skin, indicating

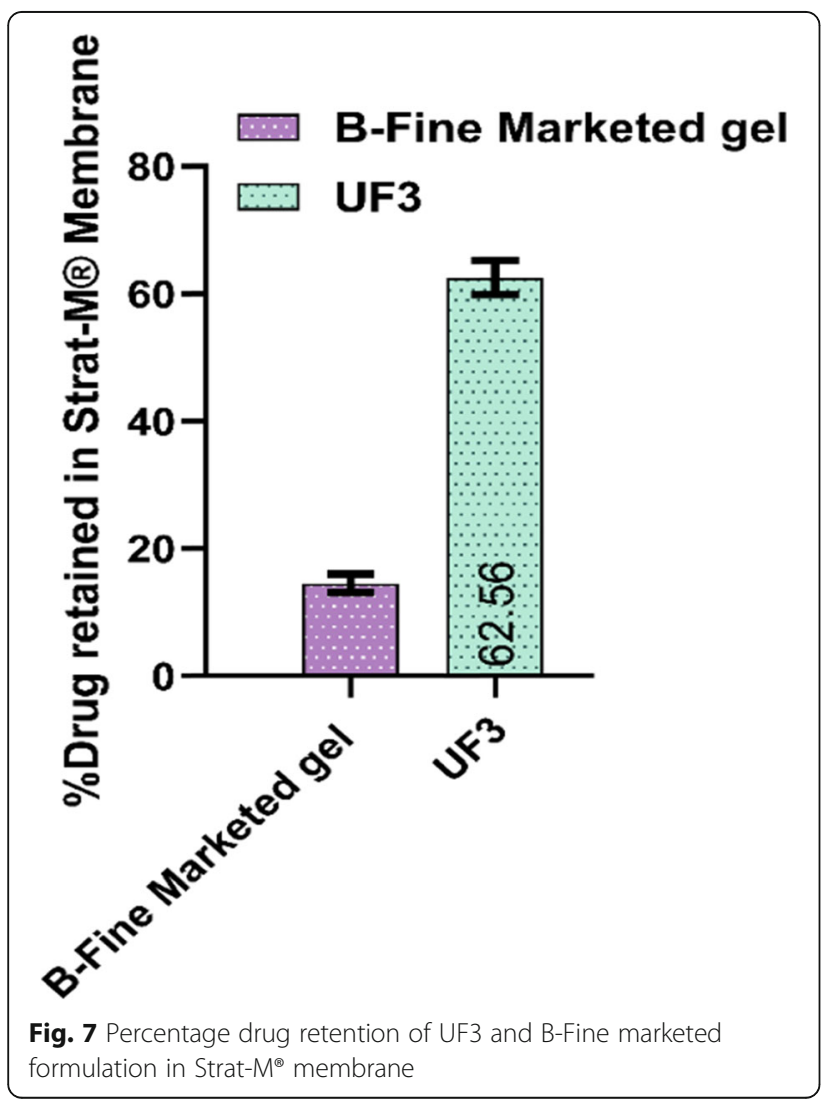

reserve ware effect of glyceryl oleate ufasomes, and therefore, therapeutic efficacy of UF3 was found to be better as compared to plain drug and B-Fine marketed drug.

\section{Discussion}

Ufasomes are unsaturated fatty acid suspensions that have lipid bilayer and a fatty acid composite [30]. So far, very few researches have been carried out in this direction. Many lipid-based formulations could be developed, i.e., liposomes, exosome, noisome, and ethosome for such studies [31]. However, ufasome formulations have many advantages than the other lipid-based formulations, i.e., ufasomes are highly penetrable as compared to similar lipid-based formulations; furthermore, as far as the entrapment efficacy is concerned, ufasomes depicts higher entrapment percentage as compared with analogous lipid-based formulations [32]. This is because the drug itself configured with lipid to form stable fatty acid vesicles. But this manuscript is entitled to provide ufasome-based drug delivery of terbinafine hydrochloride $(2 \% \mathrm{w} / \mathrm{w})$ for the treatment of Candida albicans fungal infection [33]. By utilizing the thin-film hydration method, terbinafine hydrochloride ufasomes are prepared in a round bottom flask. During the preparation process, it observed that a 3:7 ratio of drug and glyceryl oleate produces the best qualitative ufasomes. The cationic nature of optimized ufasomes (UF3) witnessed from the optimized zeta potential, i.e., $33.37 \pm 0.231$ $\mathrm{mV}$, which indicates the prepared ufasomes are having excellent physical stability with little agglomeration. From the DSC analysis, it confirmed that drugs started melting at $178.43{ }^{\circ} \mathrm{C}$. However, no such peak persisted in prepared ufasomes while analyzing the DSC graph, which indicates glyceryl oleate vesicles encapsulated drugs consistently. It was also a parameter that shows physical stability and possible higher entrapment efficacy of the prepared ufasomes. From the in vitro drug release studies, it was confirmed that a maximum $55.48 \%$ cumulative drug release was seen in UF3-optimized formulation after $30 \mathrm{~h}$ with zero-order kinetics. From the in vitro antifungal activity of applied ufasomes in animal skin, it was confirmed that UF3 was showing the highest zone of inhibition $(21.2 \mathrm{~mm})$ claimed to be an optimized formulation among all the formulations. Nevertheless, from the in vitro antifungal activity studies, it confirmed that almost all the formulations have a higher zone of inhibitions compared to the plain drug, which indicates a robust antifungal activity of the prepared ufasomes. The unique feature of this study was utilization of Strat$\mathrm{M}^{\bullet}$ membrane (Millipore, Canada) for in vitro skin permeability studies. Surprisingly, drug retention in Strat$\mathrm{M}^{\bullet}$ membrane was found to be higher as compared with the B-Fine marketed gel, which indicates prepared ufasomes had less permeation within the skin and have an 


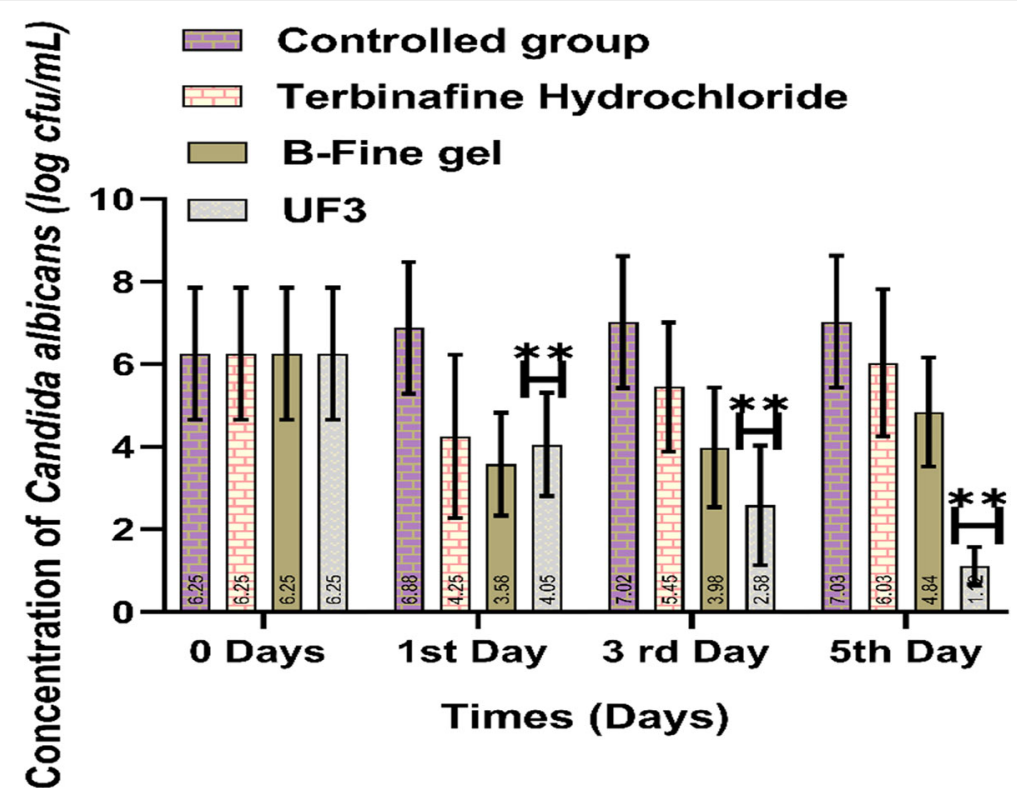

Fig. 8 In vitro antifungal activity of terbinafine hydrochloride, B-Fine gel, and UF3. Values expressed as mean \pm SD $(n=3)$, where ${ }^{* *} p<0.05$ UF3 formulation versus other formulations

excellent qualitative carrier system within. From the diffusion studies, the zero-order drug release nature of the formulations was identified, which means formulation could retain in the skin and release drug sustainability. From this study, one more thing is more prominent, i.e., the presence of glyceryl oleate in ufasomes produces reservoir effects. Finally, from the in vitro antifungal activity studies against Candida albicans, it was confirmed that optimized formulation (UF3) provides higher antifungal activity against the marketed drug. Therefore, UF3 formulations were found to be the best candidate to trigger the antifungal effect against Candida albicans-induced associated fungal infections.

\section{Conclusion}

The effect of size in drug delivery, effective drug to lipid ratio, entrapment efficacy was studied in current research. For the in vitro skin permeation and in vitro antifungal activity studies, plain drug and vesicular dispersion of drug was compared with the marketed formulation. After the induction of cutaneous candidiasis in rats, the research revealed that ufasomes of glyceryl oleate produces good antifungal activity after 5 days of study. Therefore, ufasomes were found to be more effective as compared with the marketed product. Moreover, the FU3 formulation was found to be the optimized formulation. Due to the presence of glyceryl oleate in ufasomes, it has become an excellent drug delivery carrier for any antifungal drugs. Glyceryl oleate could quickly form droplets in the skin to easily be distributed and penetrated within the skin. However, more research needs to be warranted to prepare cationic-based ufasomes for better stability. From this research, it concluded that UF3 could be effective against Candida albicans-induced associated fungal infections.

\section{Acknowledgements}

The author is like to acknowledge the help and motivation of Dr. R.S. Gaud, Director, SVKM's NMIMS Deemed-to-be University, Shirpur Campus, and Dr. Ambikanandan Misra, Director Pharmaceutical Research, Shobhaben Pratapbhai School of Pharmacy \& Technology Management, SVKM's NMIMS

Deemed-to-be University, for providing excellent research facilities and profound motivation while perusing this project.

Plant authentication

Not applicable

Author's contributions

Conceptualization, supervision, validation, visualization,writing —original draft, and writing - review and editing. The authors read and approved the final manuscript.

\section{Funding}

No funding is required. Only internal institutional funding utilized.

\section{Availability of data and materials}

Will be made available on request

Ethics approval and consent to participate

All the animal experiments were performed only after the Committee for the Purpose of Control And Supervision of Experiments on Animals (CPCSEA) prior approval [ISFCP/IAEC/CPCSEA/Meeting No.26/2020/Protocol No. 452]

Consent for publication

Not applicable 


\section{Competing interests}

The author declares that the author has no competing interests.

Received: 24 July 2020 Accepted: 18 November 2020

Published online: 07 January 2021

\section{References}

1. Hardy JD, Muschenheim C (1934) The radiation of heat from the human body. IV. The emission, reflection, and transmission of infra-red radiation by the human skin. J Clin Invest 13(5):817-831

2. McLafferty E, Hendry C, Farley A (2012) The integumentary system: anatomy, physiology and function of skin. Nurs Stand (through 2013) 27(3):35

3. Lancerotto L, Stecco C, Macchi V, Porzionato A, Stecco A, De Caro R (2011) Layers of the abdominal wall: anatomical investigation of subcutaneous tissue and superficial fascia. Surg Radiol Anat 33(10):835-842

4. Elias PM (2007) The skin barrier as an innate immune element. In: Seminars in immunopathology. Springer. Vol. 29. No. 1

5. Lefebvre CL (1934) Penetration and development of the fungus, Beauveria bassiana, in the tissues of the corn borer. Ann Bot 48(190):441-452

6. Brown GD, Denning DW, Gow NA, Levitz SM, Netea MG, White TC (2012) Hidden killers: human fungal infections. Sci Transl Med 4(165):165rv13$165 \mathrm{rv} 13$

7. Al-Jabri AA, Balkhair AA (2007) Essential immune responses to hepatitis B virus infection. Hepat B Res Adv 253

8. Garber G (2001) An overview of fungal infections. Drugs 61(1):1-12

9. Sharma VK, Sarwa KK, Mazumder B (2014) Fluidity enhancement: a critical factor for performance of liposomal transdermal drug delivery system. J Liposome Res 24(2):83-89

10. Liu JJ, Green P, Mann JJ, Rapoport SI, Sublette ME (2015) Pathways of polyunsaturated fatty acid utilization: implications for brain function in neuropsychiatric health and disease. Brain Res 1597:220-246

11. Pinilla CMB, Thys RCS, Brandelli A (2019) Antifungal properties of phosphatidylcholine-oleic acid liposomes encapsulating garlic against environmental fungal in wheat bread. Int J Food Microbiol. 293:72-78 https://doi.org/10.1016/j.ijfoodmicro.2019.01.006 Epub 2019 Jan 12. PMID: 30660071

12. Lopes NA, Pinilla CMB, Brandelli A (2017) Pectin and polygalacturonic acidcoated liposomes as novel delivery system for nisin: preparation, characterization and release behavior. Food Hydrocoll 70:1-7

13. Srisuk P, Thongnopnua P, Raktanonchai U, Kanokpanont S (2012) Physicochemical characteristics of methotrexate-entrapped oleic acid-containing deformable liposomes for in vitro transepidermal delivery targeting psoriasis treatment. Int J Pharm 427(2):426-434

14. Sharma, A., \& Arora, S. (2012). Formulation and in vitro evaluation of ufasomes for dermal administration of methotrexate. ISRN pharmaceutics.

15. Salama AH, Aburahma MH (2016) Ufasomes nano-vesicles-based lyophilized platforms for intranasal delivery of cinnarizine: preparation, optimization, exvivo histopathological safety assessment and mucosal confocal imaging. Pharm Dev Technol 21(6):706-715

16. Negi P, Sharma I, Hemrajani C, Rathore C, Bisht A, Raza K, Katare OP (2019) Thymoquinone-loaded lipid vesicles: a promising nanomedicine for psoriasis. BMC Complement Altern Med 19(1):1-9

17. Abu-Elteen, K. H., \& Hamad, M. (2011). Antifungal agents for use in human therapy. Biol Appl.

18. Ryder NS, Wagner S, Leitner I (1998) In vitro activities of terbinafine against cutaneous isolates of Candida albicans and other pathogenic yeasts. Antimicrob Agents Chemother 42(5):1057-1061

19. Rai VK, Mishra N, Yadav KS, Yadav NP (2018) Nanoemulsion as pharmaceutical carrier for dermal and transdermal drug delivery: formulation development, stability issues, basic considerations and applications. J Control Release 270:203-225

20. Nair AB, Kim HD, Chakraborty B, Singh J, Zaman M, Gupta A, Murthy SN (2009) Ungual and trans-ungual iontophoretic delivery of terbinafine for the treatment of onychomycosis. J Pharm Sci 98(11):4130-4140

21. Stano P, Luisi PL (2010) Achievements and open questions in the selfreproduction of vesicles and synthetic minimal cells. Chem Commun 46(21): 3639-3653

22. El Maghraby GM, Barry BW, Williams AC (2008) Liposomes and skin: from drug delivery to model membranes. Eur J Pharm Sci 34(4-5):203-222
23. Cevc G, Vierl U, Mazgareanu S (2008) Functional characterisation of novel analgesic product based on self-regulating drug carriers. Int J Pharm 360(12):18-28

24. Verma S, Bhardwaj A, Vij M, Bajpai P, Goutam N, Kumar L (2014) Oleic acid vesicles: a new approach for topical delivery of antifungal agent. Artif Cells Nanomedicine Biotechnol 42(2):95-101

25. Bose S, Michniak-Kohn B (2013) Preparation and characterization of lipid based nanosystems for topical delivery of quercetin. Eur J Pharm Sci 48(3): $442-452$

26. Gupta PN, Mishra V, Rawat A, Dubey P, Mahor S, Jain S, Vyas SP (2005) Noninvasive vaccine delivery in transfersomes, niosomes and liposomes: a comparative study. Int J Pharm 293(1-2):73-82

27. Haq A, Goodyear B, Ameen D, Joshi V, Michniak-Kohn B (2018) Strat-M ${ }^{\odot}$ synthetic membrane: permeability comparison to human cadaver skin. Int J Pharm 547(1-2):432-437

28. Uchida T, Kadhum WR, Kanai S, Todo H, Oshizaka T, Sugibayashi K (2015) Prediction of skin permeation by chemical compounds using the artificial membrane, Strat-M ${ }^{T M}$. Eur J Pharm Sci 67:113-118

29. Aggarwal N, Goindi S (2012) Preparation and evaluation of antifungal efficacy of griseofulvin loaded deformable membrane vesicles in optimized guinea pig model of Microsporum canis—Dermatophytosis. Int J Pharm 437(1-2):277-287

30. Naik PV, Dixit SG (2008) Ufasomes as plausible carriers for horizontal gene transfer. J Dispers Sci Technol 29(6):804-808

31. Soltani F, Parhiz H, Mokhtarzadeh A, Ramezani M (2015) Synthetic and biological vesicular nano-carriers designed for gene delivery. Curr Pharm Des 21(42):6214-6235

32. Gupta R, Agrawal A, Meraj Anjum M, Dwivedi H, M Kymonil K, A Saraf S (2014) Lipid nanoformulations for oral delivery of bioactives: an overview. Curr Drug Ther 9(1):35-46

33. Martin MV (1999) The use of fluconazole and itraconazole in the treatment of Candida albicans infections: a review. J Antimicrob Chemother 44(4):429437

\section{Publisher's Note}

Springer Nature remains neutral with regard to jurisdictional claims in published maps and institutional affiliations.

\section{Submit your manuscript to a SpringerOpen ${ }^{\circ}$ journal and benefit from:}

- Convenient online submission

- Rigorous peer review

- Open access: articles freely available online

High visibility within the field

- Retaining the copyright to your article

Submit your next manuscript at $\boldsymbol{\nabla}$ springeropen.com 